\title{
PSYCHOSOCIAL FACTORS INFLUENCING GHANAIAN FAMILY CAREGIVERS IN THE POST-OPERATIVE CARE OF THEIR HOSPITALISED PATIENTS
}

\author{
L. Aziato, PhD, Hons (Nursing and Psych), RN \\ University of Ghana \\ School of Nursing \\ Corresponding author: aziatol@yahoo.com or laziato@ug.edu.gh \\ O. Adejumo, D Litt et Phil, RN \\ University of the Western Cape \\ School of Nursing
}

\begin{abstract}
This study explored the psychosocial factors influencing family caregivers during the care of patients who had undergone surgery and were in pain. Family caregivers play key roles in the care of post-surgical patients in different cultures. However, research has not adequately explored family caregivers' experiences within the post-operative context in Ghana. An exploratory qualitative design was employed. Data collection involved individual interviews. Concurrent content analysis was undertaken and saturation of data was achieved with 12 family caregivers and 1 key informant. The participants were recruited from the surgical department of a tertiary health facility in Accra, the capital city of Ghana. The study revealed six themes that influenced family caregivers in the care of post-surgical patients: faith, fear, feeling of relief, empathy, commitment and inadequate knowledge. Health professionals should meaningfully integrate and educate family caregivers on patient care and pain management within the postsurgical context.
\end{abstract}

Keywords: family care, Ghana, post-operative pain, qualitative research, surgical nursing

\section{INTRODUCTION AND BACKGROUND}

This study explored the factors that influenced family caregivers taking care of patients with post-operative pain. Within the African setting, the family forms an important part of all facets of an individual's life. The family is considered a group of individuals who are related by blood ties, marriage or through adoption (Nukunya, 2003:48). The family unit of mother, father and children is the nuclear family and other members such as uncles and aunts form the extended family. In contemporary Ghana it is reported that the family is gravitating towards the nuclear family, with a de-emphasis on the extended 
family system. The upsurge of education and urbanisation coupled with increased cost of living has contributed to the predominance of the nuclear family system (Assimeng, 2007:74; Nukunya, 2003:48).

During illness, the family performs a significant role in ensuring that they offer the necessary assistance. The role of the family caregiver is important within the postoperative context where the effect of anaesthesia and surgery cause weakness and postoperative pain. Post-operative patients' physical and other care needs could be met by family caregivers. For example, when a patient is unable to meet his or her self-care needs, the caregiver could assist the patient with aspects of care such as bathing.

It has been reported that there are inadequate health personnel such as nurses in Ghana to meet all care needs of patients, indicating the need to involve the family in care (Ministry of Health, 2007). A study in Ghana revealed that family caregivers or relatives supported patients with breast cancer physically, emotionally and financially (Aziato, 2009:51). Other authors have also reported support provided by family caregivers in meeting patients' care needs (Fleury, Grenier, Caron \& Lesage, 2008:44). During postoperative care, family caregivers are actively involved in the care of their post-operative patients. Family caregivers are involved in cleaning the patients, running errands and purchasing medication, among other things.

The surgical experience also triggers emotional responses in family caregivers. Some authors have reported emotional reactions among family caregivers during a patient's illness (Fabiane \& Corrêa, 2007:598). The patient's health problem may trigger an emotional reaction from the family caregiver such as joy, depression, anxiety or frustration, based on the emotional ties between the patient and the relative. The surgical experience with its associated fear of death may trigger anxiety and successful surgery could result in joy in the patient's family caregiver (Davis, 2009:76). It could be inferred that the reaction to post-operative pain could be both positive and negative, because the joy associated with successful surgery could mask the emotional reaction to postoperative pain.

\section{Problem statement}

Research in the study context reported post-surgical patients experience moderate to severe post-operative pain (Aziato \& Adejumo, 2014b:1; Clegg-Lamptey \& Hodasi, 2005:63). Pain expression is shown to be influenced by an individual's socialisation from the family during childhood and this transcends pain expression during adulthood (Finnström \& Söderhamn, 2006:418). The family's influence on pain expression is deemed important to this study. It is also presupposed that the family would expect the post-operative patient to behave towards or express pain based on preconceived ideas on how the 'family' generally responds to pain. However, the concept of pain as 
an individual phenomenon is compromised if the family caregivers have preconceived ideas about pain (Pasero \& McCaffery, 2011:20).

Studies explore the role and experiences of the family in the care of hospitalised patients (Jangland, Gunningberg \& Carlsson, 2009:199). However, there seems to be lack of literature on family experiences specific to post-operative pain management. It is possible that post-operative pain management could be enhanced if family caregivers participate actively in the management of post-operative pain.

\section{Purpose}

The purpose of this study was to provide insight into the psychosocial factors that influence family caregivers as they care for their hospitalised patients who have undergone surgery and were in pain. The study was part of a doctoral study aimed at developing a context-appropriate clinical guideline for post-operative pain management in Ghana.

\section{Operational definition of terms}

- Family caregivers are informal caregivers involved in the direct day-to-day care of patients who have undergone general surgery.

- Post-operative care involves care rendered to post-operative patients in the general surgical ward after discharge from the intensive care or post-anaesthesia care unit.

- Psychosocial factors are non-physical factors such as spiritual, emotional, psychological and social factors that influence family caregivers during care of their hospitalised post-operative patients.

\section{RESEARCH METHODOLOGY}

\section{Design}

The study employed an exploratory qualitative design that sought to gain an in-depth understanding of the factors that influenced family caregivers as they cared for postoperative patients on a surgical ward. An exploratory qualitative design is suitable where little is known about the phenomenon under study and gives voice to the participants rather than using figures (De Vos, Strydom, Fouché \& Delport, 2011:297).

\section{Setting}

The study was conducted at a tertiary teaching hospital in Accra, Ghana. 


\section{Population and sampling}

The study targeted family caregivers involved in the day-to-day care of post-operative patients. The inclusion criteria were family caregivers: (1) involved in the day-today care (such as bathing, feeding, running errands and turning the patient) of their hospitalised patients who have undergone general surgery; (2) who could communicate in the English, Ga, Twi and Ewe languages; and (3) who provided a minimum of three days of direct care. Some patients were discharged after the third day, but by then the family caregivers would have had adequate experience to share. Other patients were better by the third day and no longer required personal care from their family caregivers. Exclusion criteria were family caregivers who only visited the patient and did not provide day-to-day care, those who could not communicate with the researcher, and those who provided care for less than three days.

Purposive sampling was employed and data saturation was achieved after interviewing 12 family caregivers and 1 key informant. Data saturation was determined when the interviews generated no new themes. The key informant was sampled to gain a full understanding of a theme. The principles of qualitative research allow purposive recruitment of key informants to fully develop an emerging theme. Recruitment of family caregivers was done by the first author, who explained the study to those who met the inclusion criteria.

\section{Data collection}

Data collection was done through individual in-depth interviews using a semi-structured interview guide. Guiding questions included: 'Please tell me about the role you played in your relative's recent operation?'; 'What can you say about your relative's postoperative pain?'; and 'What did you do when your relative was in pain?' Probing of participants' responses was done as necessary. The first author who collected the data is skilled in qualitative interviewing and followed up on participants' comments to gain a full understanding of emerging themes. Interviews were conducted in the English, Twi and Ewe languages according to participants' preference. The interviewer speaks all three languages fluently. Interviews were conducted at a time and venue of participants' choice. Data were collected between November 2011 and January 2012. The interviews were recorded with a digital voice recorder and later transcribed. Participants' nonverbal behaviour was recorded as field notes to provide further context to the findings.

\section{Data analysis}

Data analysis occurred concurrently with data collection. Data generated were read several times to immerse the 'self' in the participants' world. After meaning was derived from the data and themes were identified, transcripts were exported into the NVivo 9 
software. The NVivo software was used to manage the data so that sections of data were placed under their corresponding themes. The principles of content analysis (De Vos et al, 2011:402) were followed systematically, with similar codes grouped together. The researchers had previous expertise in qualitative data analysis and ensured that rigorous standards were maintained, such as systematic data management, coding and categorisation.

\section{ETHICAL CONSIDERATIONS}

The study was approved by the Ethics Committee at the University of the Western Cape in South Africa and the Ghana Health Service Ethics Committee. Approval was obtained from the hospital involved in the study and individual participants signed or thumb-printed the consent form as appropriate. Permission was obtained to record the interviews. Anonymity and confidentiality were ensured. Family caregivers were designated codes R1 to R12 (relative 1 to 12 ) and the key informant was designated RNE (retired nurse educator).

\section{TRUSTWORTHINESS}

Scientific rigour was ensured using member checking by validating emerging themes among participants. The member checking was aided by concurrent content analysis. This was used to validate transcripts and interpretations made from the data, with follow up on emerging themes to ensure their full understanding. Also, a detailed audit trail was maintained to enable other researchers to replicate the study in other similar contexts.

Interviews conducted in English were transcribed verbatim and those conducted in Twi and Ewe (local Ghanaian dialects) were transcribed into English, focusing on the meaning of participants' accounts. However, transcripts were discussed with participants to ensure that their 'true' accounts were captured. The step of translation and back translation was omitted because of financial constraints. The first author further discussed participants' stories with experts in the respective languages to confirm accurate representation, while confidentiality was ensured during the process.

\section{RESULTS}

\section{Participants' biographical data}

The ages of the family caregivers were between 18 and 60 years. There were ten females, two males and one female key informant. The ethnic background of participants included Ga, Akan and Ewe. The study included four wives, four daughters, two mothers, one husband and one fiancé. At the time of recruitment no 'son' was involved in the care 
of patients on the surgical ward. The family caregivers included four traders, one seamstress, one hairdresser, one caterer, one carpenter, one farmer, one nurse, and two were unemployed. The key informant was a retired nurse educator (RNE) with more than 20 years' clinical experience.

The psychosocial factors that influenced family caregivers in this study included six themes, namely: faith, fear, feeling of relief, empathy, commitment and inadequate knowledge.

\section{Faith}

Participants believed that God would help their patients to undergo successful surgery, recover from the effects of surgery and that pain will subside. In this study, all the family caregivers were Christians therefore this theme may not apply to different religious affiliations. This finding makes it imperative that post-operative analgesics should be given in a timely manner because the faith in God could prevent patients' relatives from reporting patients' post-operative pain:

'I reassured her by telling her that she should take it as one of her labour pains and she will be fine. She should trust in God' (R3 - daughter) and 'I was praying that God should help her so that she returns safely’ (R11 - daughter).

\section{Fear}

Family caregivers in this study were afraid that patients may not survive the surgery. Fear of death during surgery was associated with knowledge of previous surgical mortalities. Family caregivers were of the view that successful surgery was 'by the grace of God'.

I was scared because if you are not favoured by God, you will go and not return; some people have lost their lives in the theatre so I was afraid; I was so scared; but he came back successfully by the grace of God. (R7 - mother)

I was afraid she would die; because where I came from, some people went for an operation and never returned; so I was very scared. (R11 - daughter)

\section{Feeling of relief}

Family caregivers were relieved that their patients had gone through surgery successfully. Thus, because they were relieved and happy, they were not worried about post-operative pain immediately after surgery:

I came early at dawn and I met her on the ward ... I was happy. When I came and she was able to talk to me, I was relieved; when she was able to look at me, I was relieved ... I was not concerned about her pain. (R11 - daughter) 
I was not bothered about pain because I felt his life was more important and was relieved after the operation. (R7 - mother)

It could be concluded from the data that the presence of post-operative pain was not a concern to the family caregivers immediately after surgery:

I was very happy; I was very happy; I don't know; I was relieved. So far as she is alive, she can talk; she can smile, I realised that she was ok and I thank God.... in fact pain was the last thing on my mind that first day. (R8 - fiancé)

\section{Empathy}

Empathy in this study implies a sense of putting oneself in the shoes of the patient in terms of feeling or imagining the pain the patient is experiencing. The data suggested that caregivers were driven by a feeling of empathy for the post-operative patients:

The way she was shouting, you will definitely feel for her. So you put yourself in the person's shoes that if I were to be the one on the bed, how will I have felt? (R3 - daughter)

Caregivers reacted emotionally when the patients experienced post-operative pain and made efforts to ensure pain relief. Some family caregivers felt 'uncomfortable', 'worried' and 'anxious'. Hence they reassured the patients who were in pain and treated them gently during provision of physical care such as bathing. For example:

When she is in pain, I also feel uncomfortable; I am worried; I wished she is not in pain. Sometimes when it is pulling her, she shouts; if I am cleaning her, and I touch some parts of her body, she tells me she is in pain. I will say 'Oh, I am being very gentle'; then I take my time and clean and massage her more gently. (R11 - mother)

Also, R6 (a daughter attending to her father) said: 'Usually, I support him emotionally; I reassure him'. R3, a daughter caring for her mother, narrated 'the first day after surgery, she complained of severe pain and I consoled her that all will be well since it was just the first day'.

It was also reported that the feeling of empathy for patients in pain stemmed from previous sociocultural orientation that prepared caregivers for such emotional reactions to a 'suffering person'. The participants reported that they were socialised to be empathetic towards ill family members. Family caregivers indicated that a caregiver needed to understand a patient in pain and be patient when providing care. A vivid description of this phenomenon is illustrated below: 
when someone is in pain, we pamper the person; the person is suffering so you have to reassure the person and tell the person something that will make him/her relax. If you shout at the person, he can have more pains and will not be happy and can even die; so when my husband was in pain and was restless, I had patience for him and reassured him. Sometimes when I ask him to lift himself up or turn himself, he becomes angry with me and even shouts at me.... I tried to understand him and be patient with him. (R5 - wife)

\section{Commitment}

After the initial relief that the patients' surgery had been successful, family caregivers reported that they were committed to ensuring that they helped meet the care needs of patients and helped manage post-operative pain. The study found that caregivers perceived post-operative pain as pain that could be relieved by drugs and they were therefore committed to buying prescribed drugs that would relieve their patients' pain. In this regard caregivers made sure that they bought the drugs prescribed or collected the drugs covered by the National Health Insurance Scheme: 'When she was in pain; I didn't do anything about it; I just make sure I always buy the drugs prescribed so that she can have some relief from the pain' (R12 - husband) and 'I collected the drugs on the national health insurance for her and bought the others that the insurance does not cover' (R8 - daughter).

It was realised that in their commitment to ensure that prescribed drugs were purchased, some caregivers who were financially handicapped slept at the hospital premises so that they could save the money they would have used to rent a hostel:

I sleep outside on the veranda within the hospital with other family caregivers; fortunately, since we came, there has been no rain. In the morning we bath at the mothers' hostel; we use the mosquito repellent at night. I couldn't afford the hostel fee; if I pay the hostel fee, it may affect our ability to buy drugs, so I sleep outside. I have also made up my mind that whatever medicine they prescribe, I would endeavour to buy it so he won't be in pain, so I didn't want to waste the money on renting a hostel. (R5 - wife)

Family caregivers were committed to ensuring the comfort of their patients postoperatively. Thus, caregivers provided personal care, during which they ensured that they were gentle. They were also confident that the patient would tell them about any problem during the care process, rather than the nurse. The family caregiver would communicate such needs to the health professional for the appropriate intervention, which may avert any potential problems for the patient. The committed family caregiver who provided regular care for the patient served as a communication channel between the patient and the nurse: 
I am happy to clean him myself; you see that he will be more comfortable with me than another person such as the nurse and he can tell me what is worrying him; ... and I can also tell the nurses for some medicine to be given. When I clean him, he is very comfortable. (R10 - wife)

When she was operated, one morning she asked me to clean her, which I did; it was the first time of cleaning her and I couldn't refuse. Because there was nobody, I had to do it. (R12 - husband)

Some participants believed that patients preferred family caregivers to maintain their personal care. Participants thought that patients would feel depressed and sorrowful and would think they were dying if the nurses cleaned them; thus family caregivers committed to clean their surgical patients. Participants also believed that family caregivers could help clean patients because of the inadequate number of nurses in Ghana.

I came in sometimes to clean her up to help the nurse since the patients are many and the nurses are not enough. Another issue is that some patients feel depressed and sorrowful, and if nurses bath them they feel they are dying. So if we the relatives come to clean our patients, I think it is ok. (R3 - daughter)

A key informant (the RNE) gave further insight into involvement of the family in the care of patients. It was highlighted that the family caregiver, especially women, cared for the sick at home within the African context before institutionalisation of the sick. Family caregivers were committed to care for their patients and they expected nurses to demonstrate the same commitment in the care of patients. The key informant said:

In the past, we had our system of caring for the sick in Ghana which was predominantly a women affair; decision about care was taken by the family and the sick were kept at home and cared for by women. When the Europeans came with their institutional kind of nursing, nurses took full responsibility to give the support that relatives gave to the sick. It was done in such a way that the nurse as a carer in the hospital really provided that type of comfort that a patient needed from his family. Therefore, with time, the family got adjusted to the nurse taking the place of a family caregiver. In the Ghanaian tradition, it is the family member who cares for the sick. But, because the Europeans adjusted themselves to our cultural ethos, caring with all altruism - as we called nursing those days: it was the patient first. So that commitment was there; and the people saw it. So they could easily trust their patients to the nurses on the ward to care for them whilst they went home and then came during the visiting hours. But of late, caring is not the same. Some of the basic things that nurses should do, they are looking up to the patient's family to do it. (RNE - key informant) 


\section{Inadequate knowledge}

Some family caregivers did not have adequate knowledge or experience of surgery and post-operative pain management as shown in the next quotations: 'I have not seen any operated patient in the family, so I was scared and did not know what to expect' (R7 mother) and 'I didn't call the nurses when my husband was in pain because I feel that he can complain to them himself and I didn't want to worry them' (R4 - wife).

A few family caregivers were influenced by previous experience or knowledge in their care of surgical patients. For example, a family caregiver (R6) who was a nurse indicated that apart from the prescribed drugs, she purchased extra acetaminophen (paracetamol) suppositories for her father (a post-operative patient) to use when he was in pain and nurses were not immediately available to intervene. The researchers noted that this finding was not a usual practice within the context of the study. She said:

Hmm, when I come and he is in pain, I ask him if they have given him his usual medication which I know contain pain killer; ... sometimes I give him suppository; I even buy the suppository and put it in one of his drawers [bedside locker] so that when he is in pain, he sometimes inserts it himself. (R6 - daughter)

\section{DISCUSSION OF RESEARCH RESULTS}

The study reported that some family caregivers prayed for their patients so that they would undergo successful surgery, and after a successful surgery they were relieved and happy. Ghanaians have been found to believe in religio-cultural dimensions of illness (Sackey, 2002:5), and prayers by family caregivers in this study demonstrated their belief system or faith. A study among terminally ill patients indicated that faith helped family caregivers to cope (Papastavrou, Charalambous \& Tsangari, 2012:258) and family caregivers in this study found some relief after praying, as they trusted in God for successful surgery. Other studies reported that individuals in pain find relief through prayer (Mao et al, 2007:21; Shi, Langer, Cohen \& Cleeland 2007:624). This may presuppose that the individual's faith, demonstrated through prayer, is an important phenomenon within the health-care system. Perhaps future studies could explore the influence of prayer on pain management in Ghana in more depth.

Family caregivers demonstrated their empathy for the post-operative patients in pain. They indicated their understanding of pain as a consequence of surgery. The ability of caregivers to understand their patients has been identified as a cognitive component of empathy by previous authors (Davis, 2009:76). Although empathetic feelings help a caregiver to provide care to the sick more effectively (Davis, 2009:76), there were instances in this study where family caregivers expressed emotional responses to their patients' surgical experience, especially fear of death during surgery. It was realised that the surgical patients' suffering affected the emotional status of the family caregiver. This 
finding is similar to those in other cultures (Blum \& Sherman, 2010:243; Papastavrou et al, 2012:258). Thus, family caregivers within the surgical context should be aware of the effects of empathetic feelings on their own emotional states, which could negatively affect their health status if not well managed.

Participants in this study indicated their commitment to care for the surgical patients. Assimeng (2007:74) and Nukunya (2003:48) note that the family system in Ghana expects commitment to care and support from the family during times of crisis such as illness. The authors conclude from the literature that a meaningful and personal relationship between the family caregiver and patient may affect the degree of commitment to care. In this study participants stated that they provided financial, emotional and physical support to their post-operative patients. The demonstration of commitment could be linked to caregiver burden, identified by previous authors in countries such as the United States of America and Tanzania during care of patients with cancer, heart failure and HIV infection (Blum \& Sherman, 2010:243; Pallangyo \& Mayers, 2009:481). The family caregiver committed to caring for a post-operative patient could be overburdened with the care needs of the patient.

Inadequate knowledge influenced family caregivers during the care of their postoperative patients. It was realised that caregivers did not actively report post-operative pain to health professionals, as had been expected. The sometimes inappropriate 'reassurance' offered by family caregivers ('I reassured her that she should take it as one of her labour pains and she will be fine') indicated that family caregivers may not be aware that post-operative pain can be effectively managed. It can be concluded from the findings that family caregivers may also be ignorant about the complications of inadequately managed post-operative pain that could be detrimental to the patients' health; hence their encouraging the patient to bear the pain. The complications that may arise could prolong hospitalisation and lead to persistent pain and distress for both the patient and relative; as such, some nurses consider post-operative pain management a priority (Aziato \& Adejumo, 2014a:269). Although non-pharmacological approaches to pain management are recommended as adjuvant therapy (Williams, Davies \& Griffiths, 2009:145), family caregivers should give appropriate reassurance or emotional support as desired in the Ghanaian culture.

\section{CONCLUSIONS}

Post-surgical patients require family caregivers to assist in care which includes postoperative pain management. The findings of this study suggest that caregivers should be educated to appreciate the advantages of effective post-operative pain management, so that they can actively participate in pain management. Also, preoperative care should include family caregivers in order to reduce the fear and anxiety they experience. The Ghanaian culture that upholds strong family ties and relationships should be encouraged 
and perpetuated, so that caregivers continue to demonstrate commitment to the care of their loved ones. In this light, health professionals should ensure that family caregivers are assisted in their efforts to actualise commitment to care for the patients. Health professionals should create an enabling environment to promote effective family involvement in the care of surgical patients.

\section{RECOMMENDATIONS}

Health professionals should reinforce education of patients and their family on the surgical experience and post-operative pain management. It is also recognised that there is the need for policies that recognise and effectively integrate family caregivers in patient care. Health professionals, especially nurses, should demonstrate empathy and commitment to patient care. It is necessary for the health system in Ghana to accommodate the observance of faith practices for surgical patients and their families as faith influences the response to surgery.

\section{LIMITATIONS}

Lack of forward and back translation of non-English interviews due to budgetary restrictions could have affected accuracy of participants' representations in this study. The study recruited caregivers who were Christians (not purposeful), and there could be limitations with application of findings to non-Christian populations. Also, other family members such as sons, cousins, nieces and nephews may portray different perceptions, because of different family ties and degrees of relationships which may affect the commitment and care given. Therefore, future research should target such participants to establish any differences. Future studies could also measure variables such as the emotional reaction of family caregivers preoperatively and post-operatively to ascertain any differences.

\section{REFERENCES}

Assimeng, M. 2007. Social structure of Ghana: a study in persistence and change. Accra: Ghana Publishing Corporation.

Aziato, L. 2009. Post-mastectomy experiences of women with breast cancer in the Accra metropolis. West African Journal of Nursing, 20(1):51-57.

Aziato, L. \& Adejumo, O. 2014a. The Ghanaian surgical nurse and postoperative pain management: a clinical ethnographic insight. Pain Management Nursing 15(1):265-272.

Aziato, L. \& Adejumo, O. 2014b. An ethnographic exploration of postoperative pain experiences among Ghanaian surgical patients. Journal of Transcultural Nursing. http://tcn.sagepub. com/content/early/2014/05/04/1043659614526246 (Accessed 1 February 2015). 
Blum, K. \& Sherman, D.W. 2010. Understanding the experience of caregivers: a focus on transitions. Seminars in Oncology Nursing, 26(4):243-258.

Clegg-Lamptey, J.N.A. \& Hodasi, W.M. 2005. An audit of aspects of informed consent and pain relief in general surgical units of Korle-Bu Teaching Hospital. Ghana Medical Journal, 39(2):63-67.

Davis, M.A. 2009. A perspective on cultivating clinical empathy. Complementary Therapies in Clinical Practice, 15(2):76-79.

De Vos, A.S., Strydom, H., Fouché, C.B. \& Delport, C.S.L. 2011. Research at grass roots: for the social sciences and human service professions. Pretoria: Van Schaik.

Fabiane, U. \& Corrêa, A.K. 2007. Relatives' experience of intensive care: the other side of hospitalization. Revista Latino-Americana De Enfermagem, 15(4):598-604.

Finnström, B. \& Söderhamn, O. 2006. Conceptions of pain among Somali women. Journal of Advanced Nursing, 54(4):418-425.

Fleury, M.J. Grenier, G. Caron, J. \& Lesage, A. 2008. Patients' report of help provided by relatives and services to meet their needs. Community Mental Health Journal, 44(4):271-281.

Jangland, E. Gunningberg, L. \& Carlsson, M. 2009. Patients' and relatives' complaints about encounters and communication in health care: evidence for quality improvement. Patient Education and Counseling, 75(2):199-204.

Mao, J.J., Farrar, J.T., Xie, S.X., Bowman, M.A. \& Armstrong, K. 2007. Use of complementary and alternative medicine and prayer among a national sample of cancer survivors compared to other populations without cancer. Complementary Therapies in Medicine, 15(1):21-29.

Ministry of Health. 2007. Ministry Of Health, Republic of Ghana Human Resource Policies \& Strategies for the Health Sector 2007-2011. Accra: Ministry of Health, Republic of Ghana.

Nukunya, G.K. 2003. Tradition and change: an introduction to sociology. 2nd Edition. Accra: Ghana University Press.

Pallangyo, E. \& Mayers, P. 2009. Experiences of informal female caregivers providing care for people living with HIV in Dar es Salaam, Tanzania. Journal of the Association of Nurses in AIDS care, 20(6):481-493.

Papastavrou, E., Charalambous, A. \& Tsangari, H. 2012. How do informal caregivers of patients with cancer cope: a descriptive study of the coping strategies employed. European Journal of Oncology Nursing, 16(3):258-263.

Pasero, C. \& McCaffery, M. 2011. Pain assessment and pharmacologic management. St. Louis, MO: Mosby/Elsevier.

1. Sackey, B.M. 2002. Faith healing and women's reproductive health: Institute of African Studies. Sociocultural dimension of reproductive health and human development. Research Review New Series: University of Ghana, 18(1):5-11.

Shi, Q., Langer, G. Cohen, J. \& Cleeland, C.S. 2007. People in pain: how do they seek relief? The Journal of Pain, 8(8):624-636.

Williams, A.M., Davies, A. \& Griffiths, G. 2009. Facilitating comfort for hospitalized patients using non-pharmacological measures: preliminary development of clinical practice guidelines. International Journal of Nursing Practice, 15(3):145-155. 